\title{
Energy efficiency analysis of an entire ceramic kiln: A numerical approach
}

\author{
Giuseppe Cantore ${ }^{1}$, Massimo Milani ${ }^{2}$, Luca Montorsi ${ }^{2 *}$, Fabrizio Paltrinieri² \\ ${ }^{1}$ Department of Engineering “Enzo Ferrari” - University of Modena and Reggio Emilia, Via Pietro Vivarelli 10 - int. 1 - 41125 \\ Modena, Italy \\ ${ }^{2}$ Department of Sciences and Methods for Engineering - University of Modena and Reggio Emilia, Via Amendola 2, Reggio \\ Emilia 42122, Italy
}

Corresponding Author Email: luca.montorsi@unimore.it

https://doi.org/10.18280/mmc_b.870307

Received: 16 April 2018

Accepted: 20 May 2018

\section{Keywords:}

lumped and distributed parameter model, heat transfer, energy efficiency, ceramic industry, tunnel kiln

\begin{abstract}
The paper focuses on the numerical analysis of an industrial ceramic kiln to improve the energy efficiency and the fuel consumption as well as the pollutant emissions. The entire ceramic kiln is modelled by means of a lumped and distributed parameter model; particular care is devoted to the modelling of the heat transfer phenomena occurring within the system under actual operating conditions. Models for the simulation of the different components that are used for the kiln functioning are included in the modelling, such as the burners, the fan, the valves and the control system. The numerical approach demonstrates to predict accurately the temperature distribution of both the tiles and the hot gases along the kiln length. Numerical results are validated against experimental measurements carried out on a real ceramic kiln during regular production operations. The developed numerical approach demonstrates to be an efficient tool for investigating different design solutions for the kiln's components as well as for developing new control strategies. The kiln numerical model is employed to compare different configurations of heat recovery and solutions for improving the tiles' heat transfer. The considered designs are simulated and the performance in terms of energy efficiency and fuel consumption is determined.
\end{abstract}

\section{INTRODUCTION}

The ceramic industry is well known to be characterized by energy intense processes and among them, the ceramic firing in the kiln is one of the main responsible of the primary energy consumption. Recent regulations for the energy consumption limit the fuel or electricity use [1] and therefore, the design of the kiln plays a fundamental role in meeting the regulations' requirements.

Numerical simulation tools have proved to be an important instrument in the design process of the kiln and demonstrated good reliability in evaluating the complex physical phenomena that take place in industrial ceramic kilns. Different approaches have been adopted for the optimization of the kilns' performance, such as in [2] a theoretical formulation for the prediction of the kiln operating characteristics has been proposed under steady state conditions and many simplifications to the physical phenomena had to be made in order to close the mathematical model. Similar approach was adopted also in [3], in which the main focus was the assessment of the heat transfer between the hot air flow and the kiln walls. The numerical simulation becomes a valuable tool in investigating the performance of complex systems including different physical phenomena. While CFD approaches such as [4] requires a remarkable computational effort to simulate the entire systems, lumped and distributed parameter numerical analysis has been extensively adopted for the simulation of complex systems under time dependent operating conditions. The lumped and distributed parameter numerical modelling, or $0 \mathrm{D} / 1 \mathrm{D}$ approach, simulate the components of a complex system simplifying the geometry as a volume in which the quantities of the considered physics description are function of time. Eventually the variables' discretization is considered along one direction and the variation of the physical quantities in one dimension is also accounted for. In [5] a novel concept for the hydrogen production has been investigated by modelling the entire proposed plant and different operations have been compared in order to define the best configuration in terms of energy efficiency. Similarly, in [6] the numerical analysis of an entire coffee roaster plant was carried out to determine the energy efficiency of different configurations of the system while holding the same quality of the product. Nuñez Vega et al. [7] proposed a lumped parameter to simulate the convective drying process of fruits and vegetables and the optimal parameters were found. The modelling approach has also been employed for the analysis of integrated waste-to-energy systems with low environmental impact and the advantages with respect to the traditional technologies have been outlined [8].

Despite the dimensional approximation, the 0D/1D models proved also to be able to account for complex physics involving heat transfer phenomena while including the layout of the full system.

In this paper the 0D/1D modelling is adopted to analyze the performance of an entire ceramic kiln under actual operating conditions. Each component of the real kiln is accurately simulated and particular attention is devoted to predict the heat transfer phenomena that take place in the system. The results obtained by means of the proposed model are validated against experimental measurements carried out on the real facility and the numerical model is employed to evaluated different 
strategies for the kiln operations. First, the exploitation of the exhausts from a gas turbine for enhancing the heat exchange in the pre-heating section of a ceramic kiln is investigated. The effects of the exhausts injection into the kiln are evaluated in terms of both the heat absorbed by the tiles due to the increased convection phenomena and the reduction of the kiln natural gas consumption due the enhanced heat transfer in the preheating section. Furthermore, the cooling temperature profile of the tiles in the final section of the kiln is investigated and by means of the proposed numerical model, the operating conditions of the kiln for a new cooling profile are determined. In particular, a smoother temperature gradient in the fast cooling section is obtained in order to reduce the residual mechanical stresses of the product. Finally, the dynamic behavior of the kiln under unforeseen events is investigated. The influence of the burners' temperature set point on the total fuel consumption is evaluated when gaps in the production phase take place unexpectedly.

\section{MATERIALS AND METHODS}

\subsection{Numerical modeling}

The lumped and distributed parameters model of an entire real production facility is constructed by means of the LMS Imagine.LAB. AMESim ${ }^{\circledR}$ software [9]. The numerical approach simulates the compressible flow within the kiln's modules accounting for the thermo-dynamic properties of the considered gas and the semi-perfect gases approach is employed. In particular, the core of the model is realized by combining three libraries of the numerical tool: the thermal, the pneumatic, and the fluid libraries. The gases have been modeled following a gas approach in semi-perfect conditions, that is a gas that satisfies the perfect gases equation state, but is characterized by specific heat values that are function of the temperature. The solid bodies of the kiln's structure are simulated using the thermal library and each material that encompasses the kiln's components is accounted for in terms of volume, density, geometry, and thermal properties, such as the thermal conductivity and the radiation emissivity [10]. The dependence of these parameters to the temperature is also accounted for by means of polynomial expressions as in Eq. (1), Eq. (2) and Eq. (3):

$$
\begin{aligned}
& \rho=\rho_{0} *\left(1+a_{t} * \Delta T+a_{t 2} *\right. \\
& c_{p}=c_{p 0} *\left(1+b_{t} * \Delta T+b_{t 2} * \Delta T^{2}\right) \\
& \lambda_{p}=\lambda_{p 0} *\left(1+c_{t} * \Delta T+c_{t 2} * \Delta T^{2}\right)
\end{aligned}
$$

In which $\Delta T$ is the variation of temperature with respect to the reference value and the parameters and $\rho_{0}, c_{p 0}, \lambda_{p 0}$, are the respective values at the reference temperature. The numerical approach is used to study the dynamic behaviour of the gases inside the kiln under the following assumptions:

the gas flow through an orifice is mainly 1D; it is subsonic and the influence of the most relevant 2D and 3D effects is represented by introducing appropriate corrective parameters;

all the orifices have a constant and known maximum passage surface and have an infinitely short axial length;

The physical, mechanical and thermal-dynamic characteristics of the gases (density, viscosity, pressure, velocity, temperature...) can be described by their average values in space and time;

The variation of physical properties (pressure and temperature) of the gas are described following the semiperfect gases approach.

The hypotheses previously defined enable to calculate the mass flow of the fluid with the following Eq. (4):

$m=A * C_{q} * C_{m} * \frac{p_{0}}{\sqrt{T_{0}}}$

It is only function of absolute pressure and temperature at the inlet $\left(p_{0}, T_{0}\right)$, and presents two corrective parameters.

The first one, $C_{q}$, is the flow coefficient and is generally used to define the effective passage area utilized to compute the fluid velocity: for this particular application, according to the sensitivity analysis conducted by Franzoni et al. (2010), all the orifices are characterized by a flow coefficient equal to 0.95 .

The second one, $C_{m}$, is usually indicated as the "mass flow coefficient" and, respecting the subsonic flow hypotheses, is defined by the following Eq.(5):

$C_{m}=\sqrt{\frac{2 * k}{r *(k-1)} *\left[\left(\frac{p_{1}}{p_{0}}\right)^{\frac{2}{k}}-\left(\frac{p_{1}}{p_{0}}\right)^{\frac{k-1}{k}}\right]}$

where $k$ represents the isentropic expansion factor and is equal to the ratio between the specific heats of the gas, while $r$ is the characteristic constant of gases.

According to the utilization of a semi-perfect gas, the variation of pressure and temperature properties have been modeled considering both the equation of state of perfect gases and a polynomial correlation to define the variation of specific heat as a function of temperature as described in the previous equation. This approach forces a dependence of the physical properties on the temperature of the gas that is most important with respect to the pressure, but it is considered reliable only for medium-low pressures for which an instantaneous temperature variation has a greater effect on thermodynamic variables than an instantaneous pressure variation. This is exactly the situation in which the analyzed system works, with pressures close to the atmospheric one.

The Figure 1 shows the numerical model of the analyzed rollers kiln. It highlights the main sections in which the system is divided. In the modelling, the same modular design adopted in the real kiln is employed, thus, the numerical model of each module that encompasses the real facility is created and linked together as in the real kiln assembly.

In the following, the numerical models that simulate each module and component adopted in the real kiln are described in details. The modules include four main sections that accounted for the thermo-fluid-dynamics behavior of the upper and lower chambers, the tiles and the roller, see Fig. 2.

Each section of the module's numerical model includes the sub models for the description of the proper physical phenomena that take place. Particular attention is devoted to modelling the heat exchange between the kiln walls, the hot air flow, the tiles and the rollers. Conduction, convection and radiative heat transfers are evaluated for every different surface and an appropriated calculation method is adopted to compute the value of each thermal exchange between the elements of the kiln 
schematic, is a reference for the sign of the calculated quantities and it depends on the temperature difference that is determined between the considered bodies that exchange heat.

i

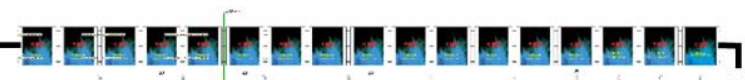

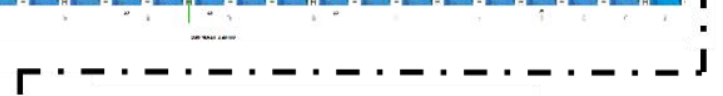

. $9^{\text {Fan\#2 }} \leq$ Fan\#3

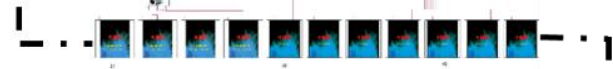

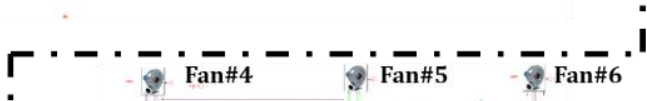

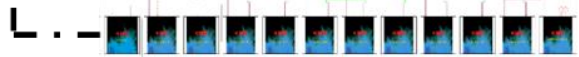

Figure 1. Layout of the numerical model of the reference ceramic kiln: a) firing section and b) cooling section of the kiln

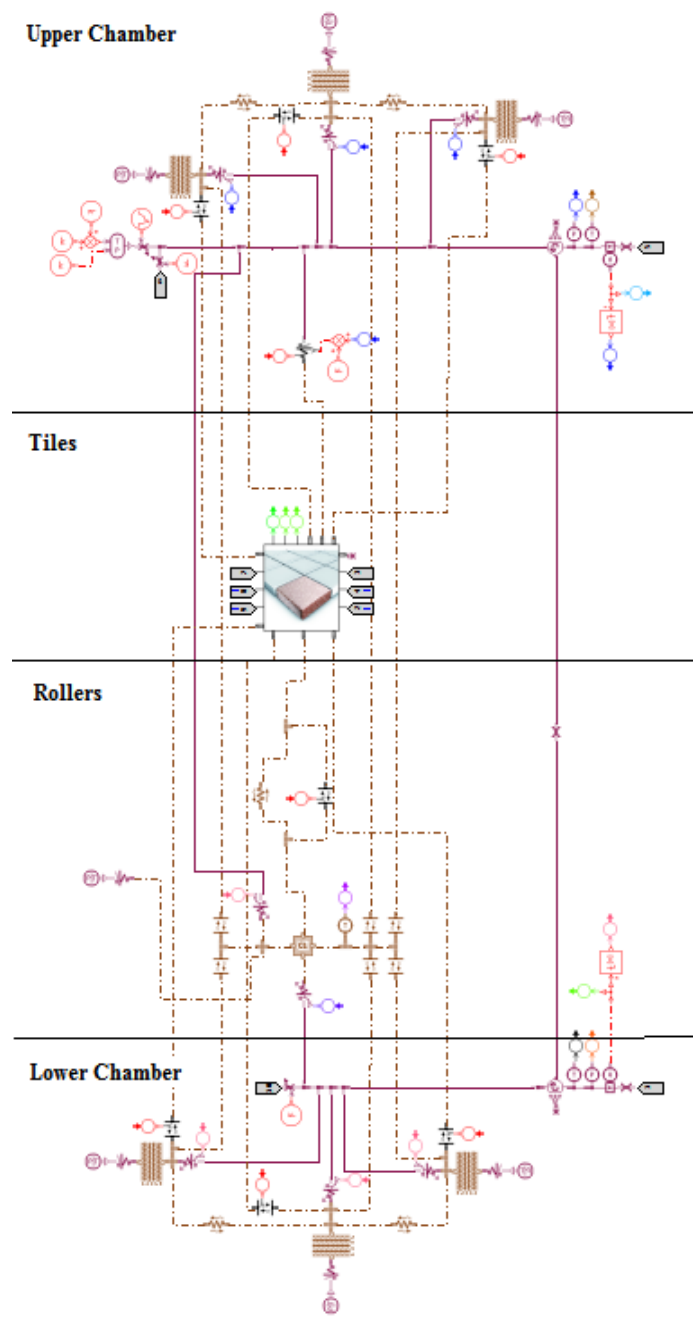

Figure 2. Main sections of the module's numerical model: upper and lower chambers, tiles and rollers

Figure 3 details the heat exchanges that are included in the module's model highlighting the type of heat transfer, such as conduction, convection and radiation heat transfer. The direction of the heat flux, i.e. the direction of the arrow in the
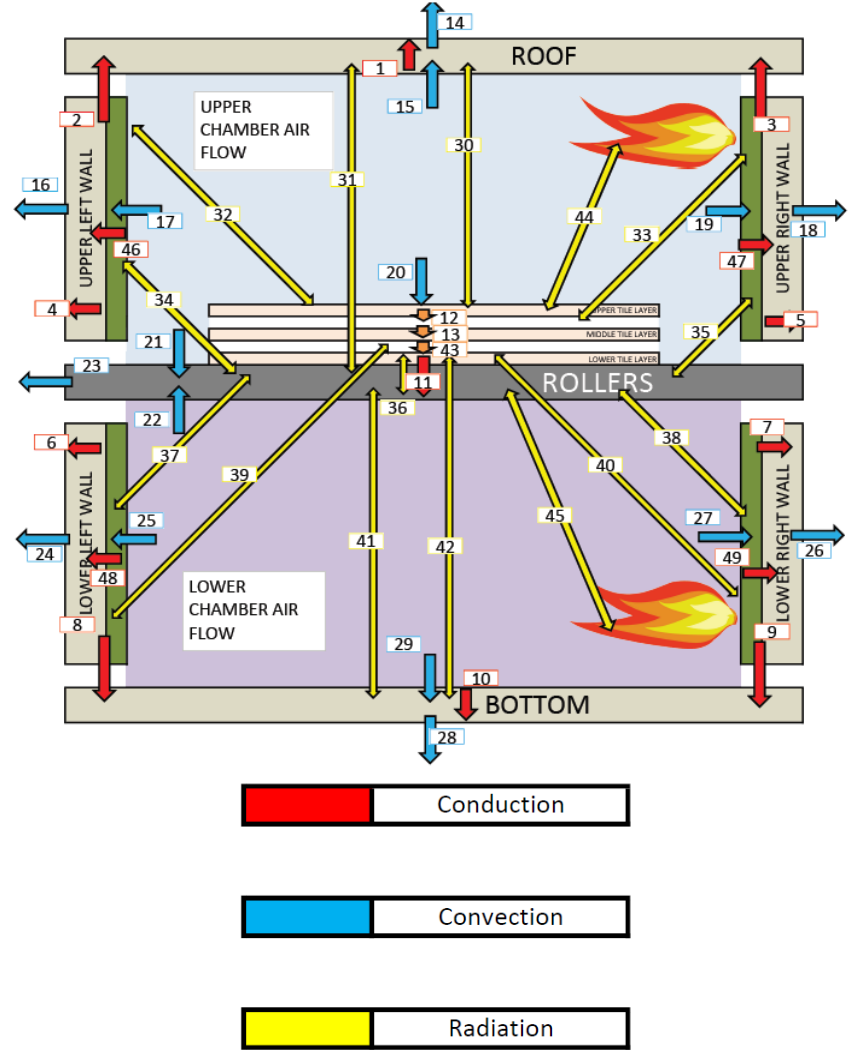

Figure 3. Schematic of the heat fluxes that characterize each kiln module' numerical models

According to the type of modules, different materials and insulation layers are accounted for, as well as the connection to external devices can be included. For instance, if burners are employed in the considered module, the interaction between the burners' flame and the ambient and the material's surfaces is accounted for.

Figure 4 shows the heat transfer types included in the modelling and the equations adopted are reported in the following.

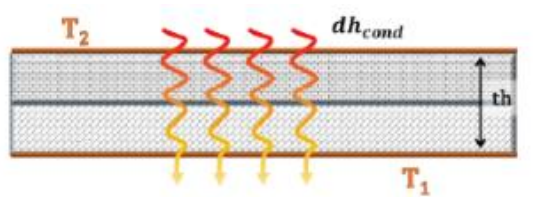

a)

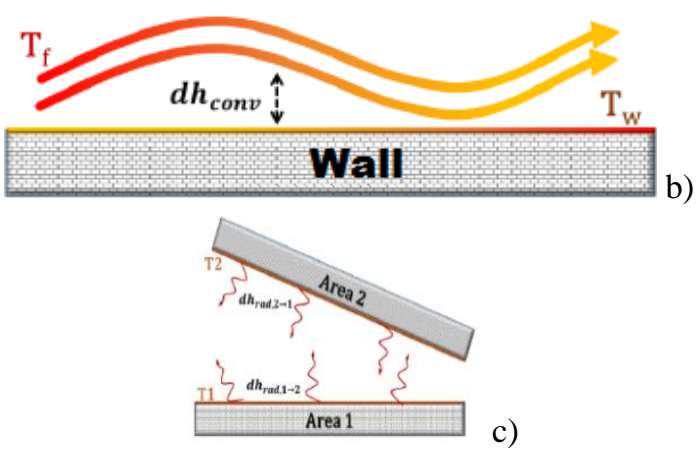

Figure 4. Schematics of the different types of heat transfer included in the modelling: a) conduction, b) convection and c) radiation 
The conduction heat transfer is computed by Eq. (6) :

$d h_{\text {cond }}=\frac{\lambda * A_{\text {cond }}}{t h} *\left(T_{2}-T_{1}\right)$

where $\lambda$ is the thermal conductivity coefficient of the material in which the thermal flow propagates, $A_{\text {cond }}$ is the thermal exchange area and th is the distance between the two sides where the reference temperatures for the calculation are defined. The convection heat transfer is calculated by means of the local Nussult number as reported in the Eq. (7):

$d h_{\text {conv }}=\frac{N u * \lambda}{c l} * A_{c o n v} *\left(T_{f}-T_{w}\right)$

where $\frac{N u * \lambda}{c l}$ is the convective exchange coefficient, $A_{\text {conv }}$ is the exchange area, $T_{f}$ is the fluid temperature, $T_{w}$ is the wall temperature, $\mathrm{Cl}$ is the characteristic length of the thermal exchange and $N u$ is the Nusselt number. In case of a mixed convection condition, the Nusselt number is calculated with the Eq. (8):

$N u=\sqrt[3]{N u_{\text {free }}^{3}+N u_{\text {forced }}^{3}}$

where $N u_{\text {free }}$ and $N u_{\text {forced }}$ are respectively the Nusselt number calculated for natural and forced convection using Eq. (9) and Eq. (10) respectively.

$N u_{\text {free }}=\left(0.825+\frac{0.327 * R a^{\frac{1}{6}}}{\left.\left(1+\frac{0.492}{P r}\right)^{\frac{9}{16}}\right)^{\frac{8}{27}}}\right)^{2}$

$N u_{\text {forced }}=0.0296 * \operatorname{Re}^{\frac{4}{5}} * \operatorname{Pr}^{\frac{1}{3}}$

The Reynolds value is calculated by the following formula in which the air velocity value is the input of the component inserted in the numerical model and computed as a function of both the duct geometry and the incoming volumetric flow rate:

$R e=\frac{\rho * v_{\text {aria }} * c l}{\mu}$

The thermal energy exchanged by irradiation between the kiln surfaces is a non-negligible component, given the high temperatures of the surfaces, and is defined by the following equation:

$d h_{\text {rad }}=\frac{\sigma *\left(T_{j}^{4}-T_{i}^{4}\right)}{\frac{1-\varepsilon_{i}}{\varepsilon_{i} A_{i}}+\frac{1}{A_{i} F_{i j}}+\frac{1-\varepsilon_{j}}{\varepsilon_{j} A_{j}}}$

in which $\sigma$ is the Stefan-Boltzmann constant, $A_{i}$ is the emitting area of body $i, A_{j}$ is the emitting area of body $j$ and $F_{i j}$ is the shape factor with which $A_{i j}$ "sees" $A_{j i}$.

For each component included in the kiln chambers model, the emitting area and the relative shape factor are have been carefully defined as a function of the real geometries and the respective portions of surface that influence the irradiation, taking into account the relationship:

$A_{i} * F_{i j}=A_{j} * F_{j i}$
Finally, the leakages between the internal volumes and the external environment have been included in the numerical model. The main contribution of the leakages is given by the space between the rollers and the kiln wall: in the numerical model, this leakage has been defined as a pneumatic connection through which cold air can enter the kiln chamber leading to a reduction of the overall efficiency. The leakages area is calculated as a function of the roller diameter increased by a coefficient $k$, as in the formula below:

tr $r_{\text {rollers }}=N_{\text {rollers }} *\left(\frac{\pi}{4} *\left(\left(D_{\text {roller }}+k\right)^{2}-D_{\text {roller }}\right)\right.$

where $N_{\text {rollers }}$ is the total number of rollers and $D_{\text {roller }}$ is the diameter of each roller. In the real kiln, the space around the rollers is filled with insulating material; in the numerical model the presence of insulating material is taken into account with a low efflux coefficient of the duct.

The main contributions affecting the tile heating process are given by the convection exchanges between the hot air flow circulating inside the kiln chamber and the flame of the burners, the conduction that occurs in the contact area between the tiles and the rollers, and finally the irradiation of the kiln walls and the rollers at high temperature. The thermal energy exchanged during these processes is analyzed using the equations previously defined. In order to calculate the temperature gradient inside the ceramic material, in the numerical model the tile is divided into three levels having the same thickness, i.e. upper layer, middle layer, lower layer, see Figure 5.

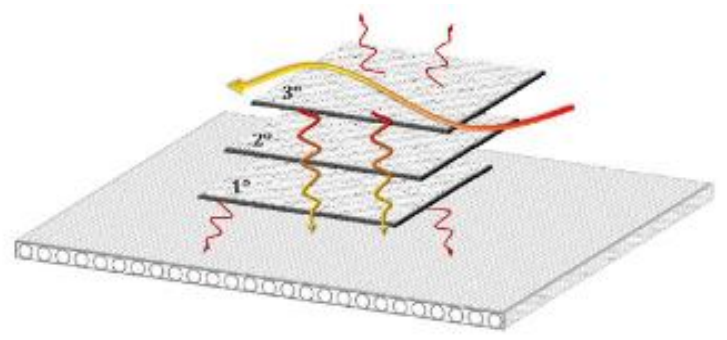

Figure 5. Schematic of the three-layer model adopted for the tiles

The first layer represents the surface of the tile in contact with the rollers and exchanges heat with the rollers by conductive and radiative phenomena. The second one is the core of the tile and exchanges heat only with the adjacent levels by conduction. Finally, the top level simulates the thermal exchanges occurring between the tile's surface and the hot air flow through the module and the burners' flame and the high temperature kiln's walls. In the analysis of the conductive thermal exchange between tiles and rollers, the global contact area is defined as a function of the external diameter of the rollers and the total number of rollers in each module as in Eq. 15:

$A r_{\text {cont }}=\frac{\left(D_{\text {roller }} * \pi * A n g_{\text {cont }}\right)}{360} * L_{\text {roller }} * N_{\text {rollers }}$

where $D_{\text {roller }}$ is the diameter of each roller, $A n g_{\text {cont }}$ is the contact angle between tiles and rollers, $L_{\text {roller }}$ is the length of each roller and $N_{\text {rollers }}$ is the total number of rollers.

The effects of the tiles' motion on the heat transfer is also considered in the modelling. Since it is not possible to define a continuous flow for a solid material, an ad-hoc model is 
created modelling the tiles as a fluid having the same thermal properties of the real tiles and flowing along the kiln with the real tiles' velocity. Therefore, the effects of the temperature gradient of the tiles in each modules can be correctly predicted.

The standard natural gas-fueled burners are simulated as a thermal power input to the upper and lower chambers of the modules according to the instantaneous fuel consumption determined by the kiln control system as a function of the temperature set point for each module. The same PID controller employed in the real kiln to regulate the temperature in the chambers and thus the natural gas flow rate for the burners is also adopted in the kiln numerical model. Therefore, the total consumption of natural gas is one of the main results of the numerical approach and it is determined as a function of the thermal balance of the kiln chamber to maintain the desired temperature in the selected modules as in the real kiln.

This energy balance is calculated for each volume representing the kiln chambers along with the continuity equation that calculates the mass balance of each module. The mass balance accounts for the flow through the modules as well as for other contributions such as the leakages and the combustion products from the burners. Particular attention is devoted to the effects of turbulence caused by the burners flame on the air flow circulating in the kiln chambers. In the modules equipped with burners, the heat exchange on the tile surface, the ceramic rollers and the walls is influenced by the speed of the flame from the burners' nozzles. The turbulence affects significantly the convective heat exchange in particular in the areas directly reached by the combustion gases. Hence, in the firing zone of the kiln, the convective coefficient is computed not only according to the average speed of the hot air flow, but also considering the velocity of the combusted gases whose value is derived from multi-dimensional simulations in those regions.

The models for different types are taken into account and an ad-hoc model for the recuperative burner type, i.e. the EKO burner, is created. The recuperative burner preheats the fuel and the oxidizing air by means of a hot air flow taken directly from the module's chamber, see Figure 6. The hot air flow is drawn from the chamber by a proper fan and its flow rate is controlled in order to have almost constant temperature of the fumes after the heat recovery. The developed numerical model of the EKO burner reproduces accurately the geometry of the ducts of the three flows that characterize the component, i.e. the fuel, the oxidizer and the hot air flow from the kiln, in order to simulate the exchange areas and the relating heat transfer coefficients.

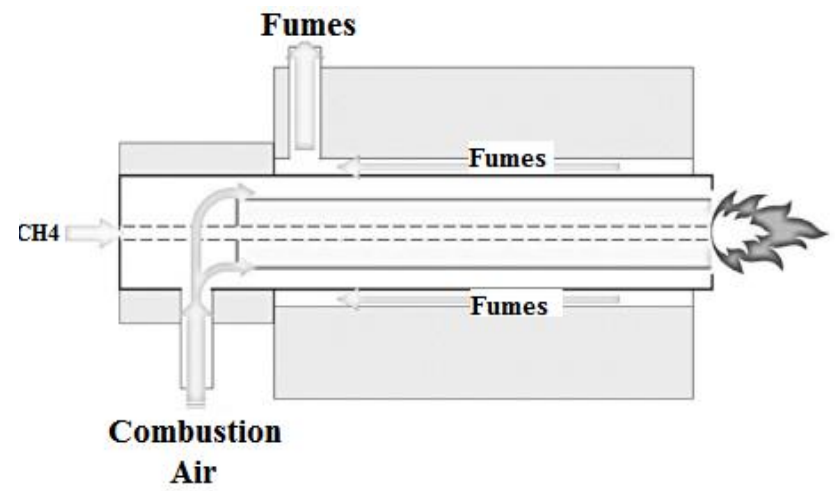

In order to predict the mass flow rates in the different sections of the kiln, the fans used to operate the system are modelled according to the operating maps provided by the manufacturers. As in the real kiln, the fan are regulated by varying the rotational speed and the mass flow rate is determined as a function of the resulting pressure drop. Finally, the many valves employed for controlling the flows in the real system are modelled as orifices. Their effective areas is determined according to the valve opening position and it can be varied to simulate different operating conditions.

\subsection{Numerical results validation}

The prediction capabilities of the kiln numerical model are validated by comparing the calculated results against the experimental measurements carried out on the real ceramic facility during regular production operations. The tiles produced during the tests had dimensions of $1.2 \times 1.2 \mathrm{~m}$ and height equal to $9 \mathrm{~mm}$ and the kiln is run with a production rate of approximately $5,000 \mathrm{~kg} / \mathrm{h}$. The gas temperature in each module of the firing section is measured with a PT 100 thermocouple, while in the cooling section the gas temperature is usually not measured. In addition, measurements using DATAPAQ ${ }^{\circledR}$ instrument were carried out in order to have a redundant measure of the air in the upper chamber of the kiln's modules and an estimate of the temperature of the tiles' surface. The instrument is a thermally insulated box equipped with a data logger connected to 8 thermocouples and it travels along the kiln on a tile's top and measures the temperature of the upstream tiles and air flow. The temperature values are measured with an accuracy of $+/-0.3{ }^{\circ} \mathrm{C}$. Figure 7 a) shows the temperature profile of the gases within the kiln upper and lower chambers and the comparison between the measured and calculated values of the temperature, the set point temperature controlled by the process is also plotted. The data are divided by a reference temperature value in order to have non-dimensional quantity. The agreement between the numerical results and the experimental measurements is satisfactory and the trend of the temperature distribution of the hot air flow along the kiln length is correctly predicted. The proposed numerical approach proved also to predict accurately the tiles' temperature along the kiln axis, see Figure $7 \mathrm{~b}$ ).

This result was particularly important, since no data were available for most part of the cooling section, due to a damage occurred to the measurement system after module 34 because of the high temperatures.

A good agreement between the experimental measurements and the calculations proved was also found in terms of natural gas consumption. The predicted fuel rate necessary to power the kiln during regime operation was calculated within a 5\% error with respect to the measure kiln fuel consumption, see Figure 8 a). Similar accuracy was found for the mass flow rate through the main fans. The calculations are compared to the experimental values and the percentage error proved to be lower than $6 \%$.

Once the numerical model is validated against experimental measurements, simulation can be employed to widen the analysis of the kiln operation by investigating quantities that are difficult to be measured and to explore novel concept for the energy efficiency enhancement and new control strategies.

Figure 6. Schematic of the recuperative burner type 


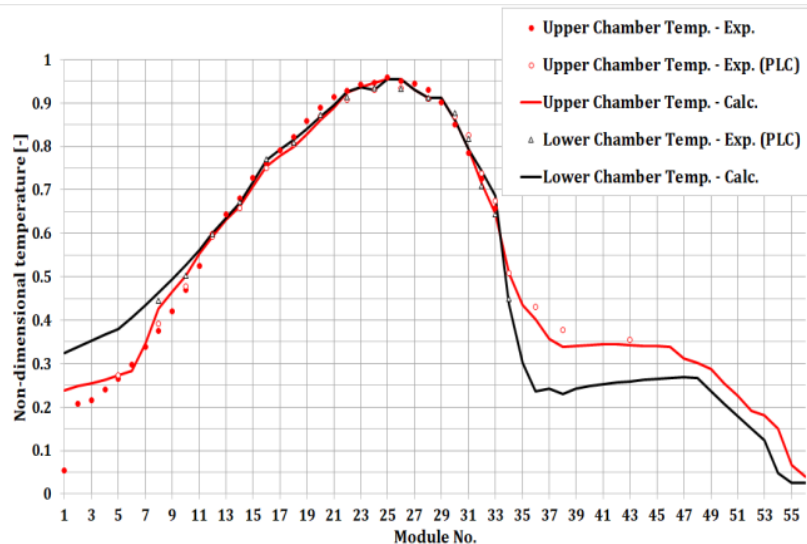

a)

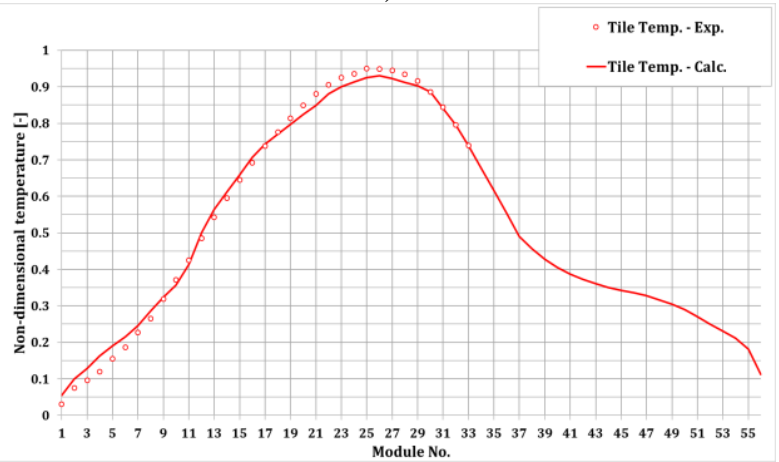

b)

Figure 7. Comparison between the measured and calculated values of the non-dimensional temperature profile along the kiln axis of a) the air in the kiln upper chambers and b) tiles top surface

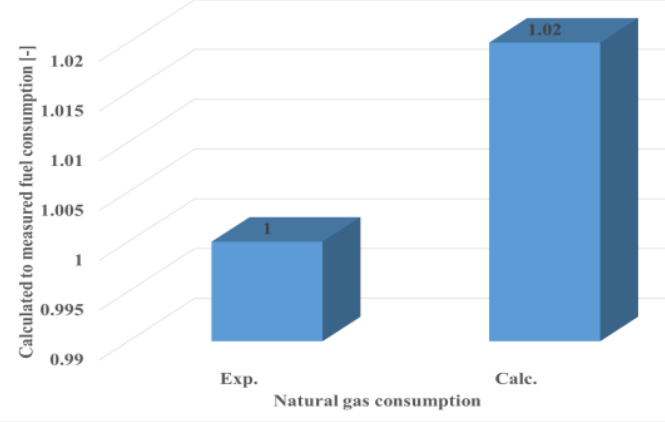

a)

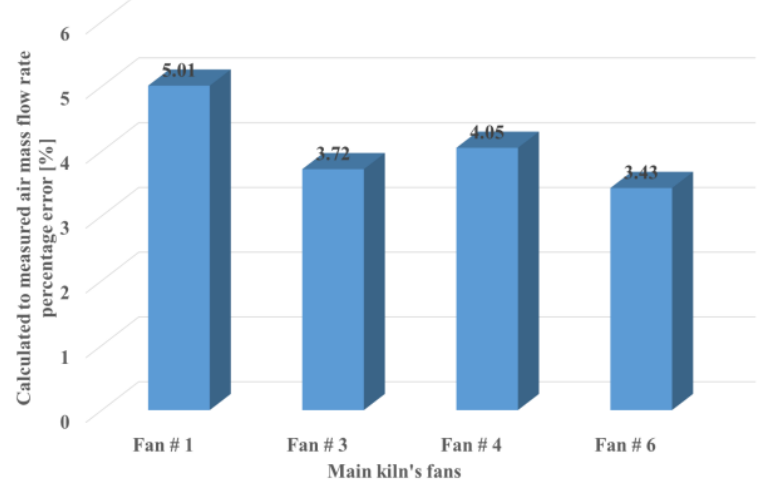

b)

Figure 8. a) Calculated to measured ratio of the natural gas fuel consumption of the entire kiln and b) calculated to measured air mass flow percentage error through the main kiln's fans

\section{RESULTS AND DISCUSSIONS}

The 0D/1D model described in the previous section is adopted to analyze the performance of the entire ceramic kiln under different strategies for the kiln operations optimization. First, the influence of the exhausts from a gas turbine for enhancing the heat exchange in the pre-heating section of a ceramic kiln is investigated. Afterward, a new cooling temperature profile of the tiles in the final section of the kiln is investigated and the relating operating conditions of the kiln are determined. Finally, the dynamic behavior of the kiln under unexpected events is investigated and the fuel consumption under stand-by operations is calculated as well as the required cool-down and warm-up time periods.

\subsection{CHP turbine unit exhaust gases exploitation}

For the simulation of the gas turbine exhausts injection into the kiln, the numerical model the upper chamber of module \#4 is modified in order to account for the pipes that blows the exhaust gases on the tiles' top surface with a temperature close to $270^{\circ} \mathrm{C}$. The number of pipes is calculated in order to have an exhaust gases outlet velocity from the blower tubes equal to approximately $15 \mathrm{~m} / \mathrm{s}$. This value is remarkably larger than the average velocity of the main hot air flow, which is close to $3.5 \mathrm{~m} / \mathrm{s}$. Therefore, the exhausts aim at enhancing the turbulent flow field on the tiles' top surface and thus to increase the convective heat transfer from the air stream and the ceramic material.

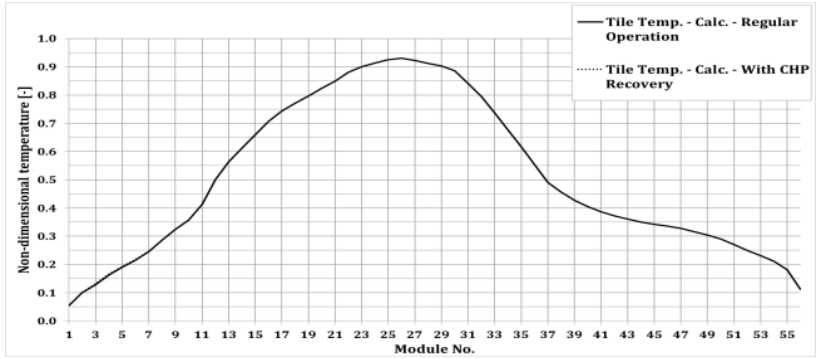

a)

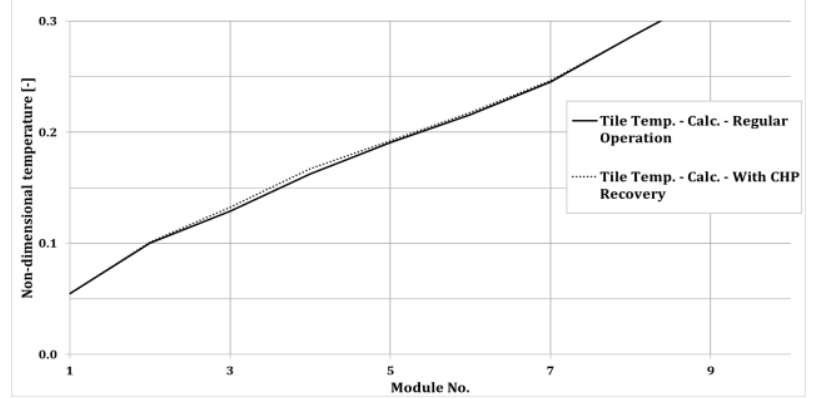

b)

Figure 9. Effects of the exhaust gases recovery from the CHP unit on the non-dimensional tile temperature profile a) along the entire kiln and b) zoomed view on the preheating modules

Figure 9 shows that the exhaust gases recovery from the CHP unit enhances the tiles' temperature profile in module \# 4 due to the enhanced turbulence. The advantage is also visible in the downstream modules with regards to the air flow rate, i.e. module \# 3 and module \# 2. The results is obtained even though the injected exhaust are slightly colder than the air stream within the kiln in modules \# 4; in fact, Figure 10 
highlights that the temperature in the upper chamber decreases in module \# 4, while it returns to the values obtained during regular operation, due to the hotter flow coming from the lower chamber. The better heat transfer in the preheating section determines a reduction in terms of natural gas consumption of the first firing module. In fact, it can be assumed that the increased amount of heat absorbed by the tiles in modules \# 4 and \# 3 has not to be provided to the firing modules in order to reach the tiles' set point temperature. This effect is clearly noticeable in module \# 12, i.e. the first firing module, where the percentage fuel consumption saving reaches the $8 \%$ for the burners in the upper chamber and $3 \%$ for the lower chamber's ones, see Figure 11. Nevertheless, the benefit reduces significantly already in the subsequent module with respect to the tile motion, i.e. module \# 13 and no saving is calculated for the farther modules, thus, the percentage reduction of the fuel consumption of the entire kiln is limited to $0.4 \%$.

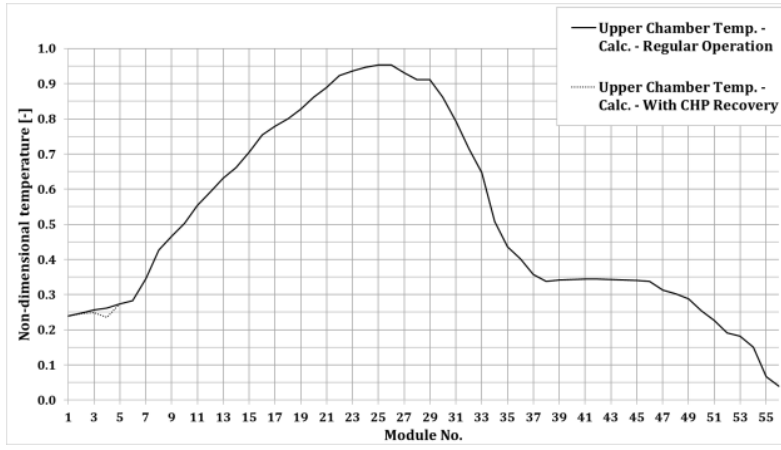

a)

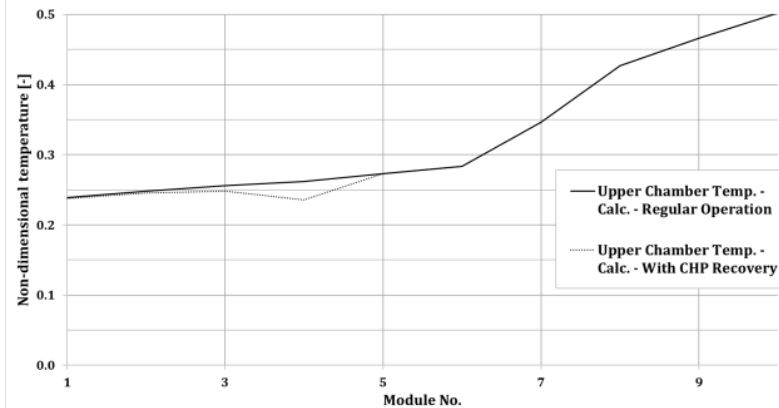

b)

Figure 10. Effects of the exhaust gases recovery from the CHP unit on the non-dimensional upper chamber air temperature profile a) along the entire kiln and b) zoomed view on the preheating modules

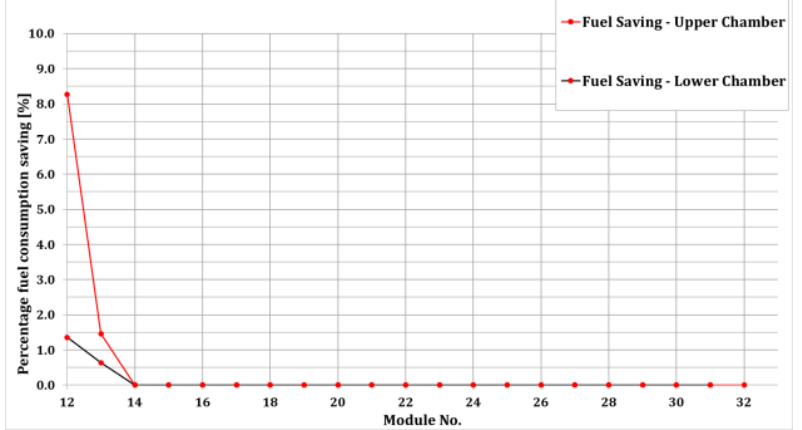

Figure 11. Effects of the exhaust gases recovery from the CHP unit on the percentage fuel consumption saving in the firing modules

\subsection{Transient behavior}

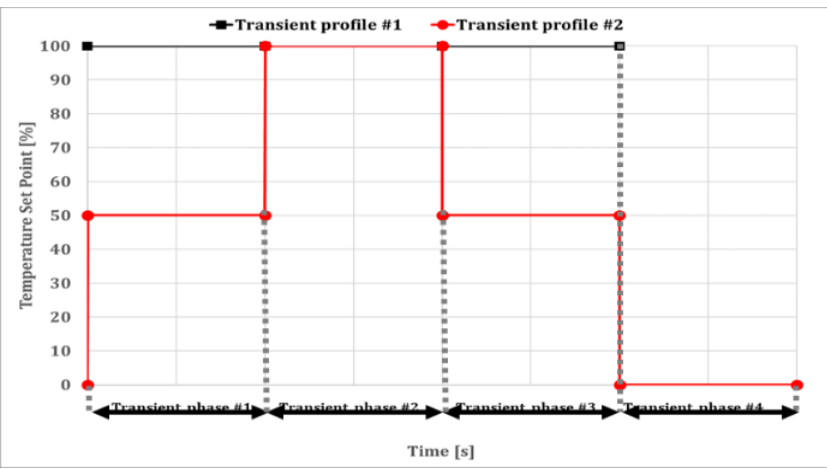

Figure 12. Time history profiles of the temperature set points for the burners' control considered in the analysis

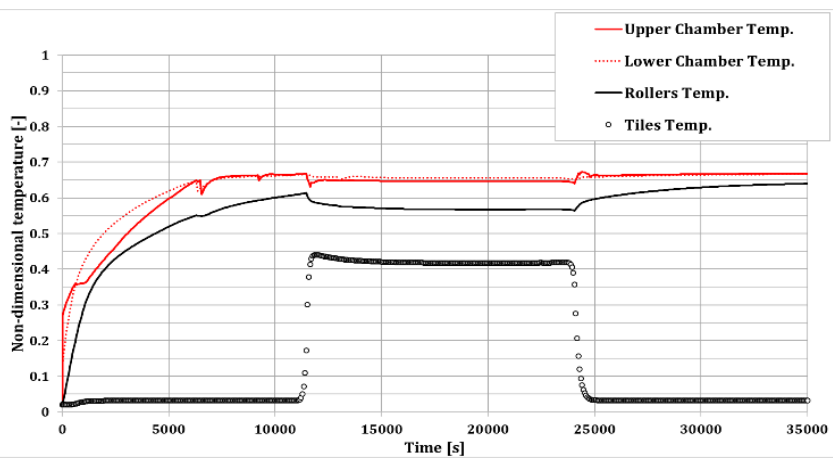

(a)

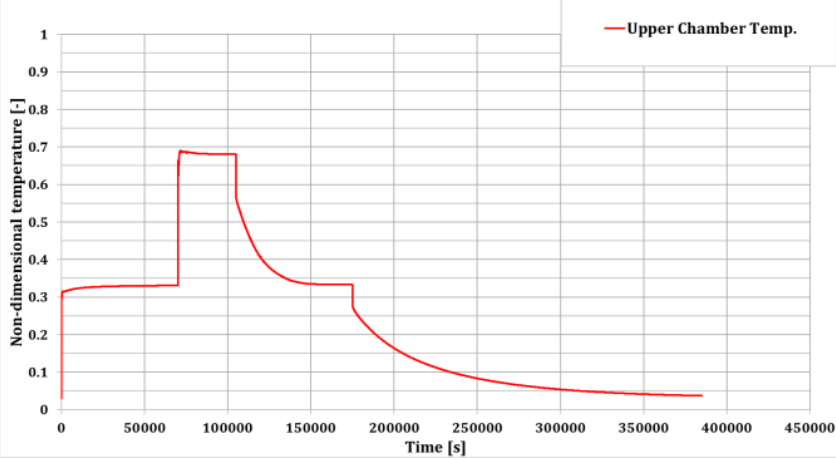

(b)

Figure 13. Time history profiles of the temperatures in module \# 10 for a) the transient profile \#1 and b) the transient profile \#2

The dynamic behavior of the kiln under unexpected events is investigated, in particular the warm-up and cool-down phases are investigated in order to address the kiln fuel consumption during transient. The numerical analysis of the kiln dynamic behavior can be used to determine in case of unexpected events during production if the standby mode operations of the kiln is more efficient rather than its complete shutdown. Figure 12 shows the simplified strategies for investigating the kiln dynamic behavior. In transient profile \#1, the temperature set points for the burners are controlled in order to have the reference temperatures for each module, i.e. temperature set points equal to $100 \%$, but during the transient phase \# 1 and \# 3 there are no tiles in kiln and only during the second transient phase the ceramic material is travelling along the systems. Figure 13 a) shows the time histories of the main temperature profiles in the module \# 10. It can be noticed how the tiles influence the temperature of the rollers: when the kiln 
is empty the radiation contribution to the rollers is much higher determining a larger temperature value. Furthermore, the rollers demonstrate to have the largest thermal inertia with respect to the hot air flow and the ceramic material.

A second numerical analysis of the transient behavior of the kiln is investigated focusing on the empty kiln operations, i.e. transient profile \#2 in Figure 12. The temperature set points for the burners in the different modules are firstly operated at the $50 \%$ of the nominal values until steady state regime is reached. Afterword, the nominal temperature set points are adopted until regime conditions are met again; finally, the shutdown phase is subdivided into two transient periods, i.e. a stand-by phase at $50 \%$ of the temperature set points followed by a complete shutdown of the burners until the kiln is cooled down to ambient temperature. The ceramic product is not considered for this analysis and the kiln operation is analyzed during production gaps. Figure $13 \mathrm{~b}$ ) shows the transient profile of the air temperature in the upper chamber of module \# 10. It can be noticed that the cool down transient of the kiln results larger than the warm up phase by several times.

\section{CONCLUSIONS}

In this paper the $0 \mathrm{D} / 1 \mathrm{D}$ model of an entire ceramic kiln is constructed and employed to simulate the performance of an entire ceramic kiln under actual operating conditions. Each component of the real kiln is accurately simulated and particular attention is devoted to predict the heat transfer phenomena that take place in the system. The numerical results have been validated against experimental measurements carried out on the real facility and the prediction resulted in good agreement with the measurements. The numerical model was then adopted for investigating different strategies that can be used to enhance the energy efficiency of the kiln. First, the exploitation of the exhausts from a gas turbine for enhancing the heat exchange in the pre-heating section of a ceramic kiln were investigated. The effects of the exhausts injection into the kiln were evaluated in terms of both the heat absorbed by the tiles due to the increased convection phenomena and the reduction of the kiln natural gas consumption.

The use of the exhausts from the CHP turbine unit resulted in a lower natural gas fuel consumption of approximately $0.35 \%$ of the entire kiln fuel requirements. Furthermore, the cooling temperature profile of the tiles in the final section of the kiln was investigated and the operating conditions of the kiln for a new cooling profile were determined. A smoother temperature gradient in the fast cooling section was obtained in order and the relating fans' speeds, valves' positions and burners' temperature set points were determined. Finally, the dynamic behavior of the kiln under unforeseen events were investigated and the characteristic time for the kiln warm-up and cool-down were calculated for different temperature set points of the firing modules.

\section{ACKNOWLEDGMENT}

The present work is part of Design for Resource and Energy efficiency in CerAMic Kilns (DREAM) project. The project has received funding from the European Union's Horizon 2020 research and innovation programme under G.A. No 723641

\section{REFERENCES}

[1] Agrafiotis C, Tsoutsos T. (2001). Energy saving technologies in the European ceramic sector: a systematic review. Applied Thermal Engineering. 21: 1231-1249. https://doi.org/10.1016/S13594311(01)00006-0

[2] Kaya S, Mançuhan E, Küçükada K. (2009). Modelling and optimization of the firing zone of a tunnel kiln to predict the optimal feed locations and mass fluxes of the fuel and secondary air. Applied Energy 86: 325-332. https://doi.org/10.1016/j.apenergy.2008.04.018

[3] Mezquita A, Boix J, Monfort E, Mallol G. (2014). Energy saving in ceramic tile kilns: Cooling gas heat recovery. Applied Thermal Engineering 65: 102-110. https://doi.org/10.1016/j.applthermaleng.2014.01.002

[4] Jiang X, He B, Liu Q, Wang D, Xu J, Xie W. (2017). A CFD analysis of gas plume stratification in confined space. Journal of Modelling, Measurement and Control B 86(2):

557-568. https://doi.org/10.18280/mmc_b.860217

[5] Milani M, Montorsi L, Paltrinieri F, Stefani M. (2014). Experimental and numerical analysis of the combustor for a cogeneration system based on the aluminum/water reaction. Energy Conversion and Management 87: 12911296. https://doi.org/10.1016/j.enconman.2014.03.027

[6] Bottazzi D, Farina S, Milani M, Montorsi L. (2012). A numerical approach for the analysis of the coffee roasting process. Journal of Food Engineering 112(3): 243-252. https://doi.org/10.1016/j.jfoodeng.2012.04.009

[7] Nuñez Vega AM, Sturm B, Hofacker W. (2016). Simulation of the convective drying process with automatic control of surface temperature. J. Food Eng. 170:

16-23. https://doi.org/10.1016/j.jfoodeng.2015.08.033

[8] M Milani, L Montorsi, M Stefani. (2014). An integrated approach to energy recovery from biomass and waste: Anaerobic digestion-gasification-water treatment, Int. Journal of Waste Management and Research 32(7): 615625. https://doi.org/10.1177/0734242X14538307

[9] LMS AMESim User Manual, 2016 Siemens Industry Software NV.

[10] Milani M, Montorsi L, Stefani M, Saponelli R, Lizzano M. (2017). Numerical analysis of an entire ceramic kiln under actual operating conditions for the energy efficiency improvement. Journal of Environmental Management 203: https://doi.org/10.1016/j.jenvman.2017.03.076 